\title{
Microcomputer graphics in psychological research
}

\author{
RONALD H. OLCH and STANLEY E. HECHT \\ Southern California Research Institute, Los Angeles, California 90045
}

\begin{abstract}
This paper discusses a methodology for psychological instrumentation in which requirements common to a spectrum of experiments are defined and implemented in a microcomputer system. The microcomputer may either stand alone or be used as an intelligent front end to a larger computer. In either case, experiments are described in a high-level implementation language. The advantages of this approach include reduced hardware and maintenance costs, increased reliability, and smoother operation by research personnel. A tutorial example is given of the design and construction of one such system used to present visual stimuli, including text, graphics, and simple animation, on a movable array of video monitors. Displays are produced by a Z80 microcomputer in response to commands from a larger host computer, in this case a PDP Lab/8e. Illustrations are given of three studies in progress; comparisons are made with special-purpose equipment built earlier. The microcomputer system is preferable from both a cost and management point of view.
\end{abstract}

A great deal of effort in psychological research is devoted to the development of instrumentation. Not only may digital computers be involved, but also specialized front-end equipment such as function generators, stimulus displays, and response devices of various types. When such equipment is developed on an experimentby-experiment basis, management difficulties may arise in the form of (1) costs and/or time delays in building hardware for each new experiment and in developing the necessary time-critical software to support the experimental paradigm, (2) the need to train personnel in the operational details of the additional hardware/software configurations, and (3) costs of system maintenance at a level sufficient to assure reliable operation. These difficulties may be particularly acute in laboratories with several studies in progress concurrently.

Analogous problems of software development have been approached by the introduction of high-level experiment languages such as SKED. The details of timing and task scheduling are then contained in a single general-purpose program, leaving the user free to implement new experiments (or modify existing ones) with little additional programming.

Thanks to the rapidly expanding market in microcomputer system components, it is now possible to take a similar approach to the problem of instrumentation. Rather than designing special-purpose hardware for each application, a microcomputer system may be assembled which implements features common to a spectrum of experimental needs. The microcomputer may either stand alone or serve as an intelligent front end to a larger computer system; configuration depends largely on experimental needs and on existing computer facilities in the laboratory. In either case, many related experiments may be run on the system without need for additional hardware development. Experiments are described in a high-level language, converted into a form suitable for interpretation by the mini/microcomputer system, and controlled by simple user commands.

The purpose of this paper is to provide a tutorial example of how a microcomputer system is designed and built for use in a human factors research laboratory. The system provides a wide range of visual stimuli, including text, graphics, and rudimentary animation. Measures of subject performance include responses on a four-way pushbutton pad, pressure on a force stick, and eye-point-of-regard (EPR). While these measurements are specific to one family of experiments, generalizations to other applications are straightforward.

\section{Background}

Many of the design criteria for the microcomputer system were developed through experience with an earlier device. The Stimulus Programming System (SPS) was built by a subcontractor (Perceptronics, Inc.) in 1975 at a cost of $\$ 31,750$. The SPS, shown in Figure 1, is still in use for divided-attention experiments. A 180-deg display arc contains a matrix of 24 digit positions. Participants sit at the center of the arc with eyes directed at the vertical midpoint. The 24 numerals are presented by red seven-segment LED displays located at 5 and 10 deg above and below the midline, and at 10,15 , and 20 deg to the right and left of center. In a typical application, all displays are lit when a trial is initiated, and then randomly selected digits change every few seconds. Whenever a " 2 " appears, the participant is required to respond as rapidly as possible using a four-way lever in the left hand to indicate the quadrant in which the numeral has appeared. At the same time, 


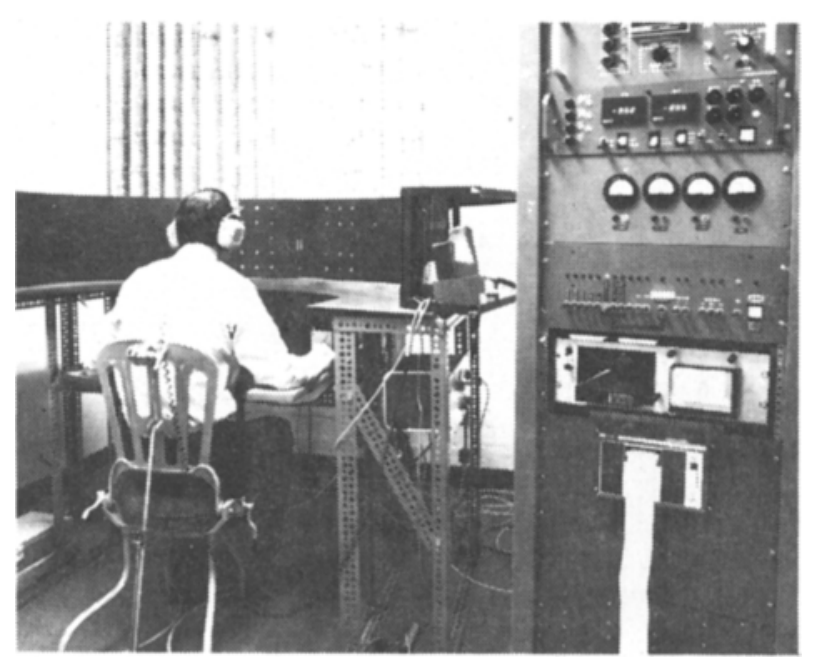

Figure 1. Stand-alone system for divided-attention experiments built in 1975 .

the right hand may operate a force stick whose output is added to a random noise signal and displayed on a Borroughs gas-discharge bar graph display in the center of the visual field. The forcing function is white noise filtered so as to roll off at $40 \mathrm{~dB} /$ octave above a switchselectable cutoff frequency $(.1, .2$, or $.5 \mathrm{~Hz})$.

Though a microcomputer is employed in this device, it is not directly programmable. A control program in memory reads and interprets symbolic instructions punched on paper tape, sequences LED presentations, and scores responses. Output is on fanfold paper, 2.25 in. $(5.7 \mathrm{~cm})$ wide.

The experiments run with the SPS are limited to those with visual stimuli of fixed size, located at a limited set of points within the visual field, and generated with a seven-segment LED or on-off lamp.

Problems have arisen in training operators because of the awkwardness of system checkout and setup. Many front panel switches, needed to set microprocessor control bits, have logical functions difficult to explain to an individual who has no background in processor design.

An extremely time-consuming step of manual data reduction and transcription from the printed paper tape was required. No provision was made for convenient digital recording of analog or discrete signals.

Furthermore, hardware malfunctions led to long periods of downtime because of the lack of modular components and quickly replaceable spares. These problems were considered as examples to be avoided while designing the microcomputer graphics system.

\section{SYSTEM DESIGN}

\section{Requirements}

As a minimum, the target system was intended to duplicate the capabilities of the SPS while relieving some of the difficulties inherent in the system. Beyond that, ease of specifying and running new experiments was stressed. The following criteria were considered desirable:

Visual field adaptable in shape

Flexible visual presentations

Text/graphics of varying size

Rapid changes of significant display areas

Rudimentary animation

Range of brightness levels

Spectrum of experiments on existing hardware

Minimal programming for new experiments

Ease of operation

No special environment or preventive maintenance

High utility, as measured by mean time between failures (MTBF) and mean time to repair (MTTR)

\section{Specification}

An array of video monitors was selected as the display medium. The use of video allowed control over factors such as brightness and size of the material presented, and the use of a number of screens permitted the shape of the visual area to be easily changed. Use of several small monitors, in this case measuring 9 in. $(22.9 \mathrm{~cm})$ diagonally, increased the expected reliability due to redundancy of parts. Overall resolution was improved by the larger number of scan lines available with multiple video channels.

A video terminal interface manufactured by Polymorphic Systems was chosen as the display generator. This unit is compatible with the S-100 bus and appears to the microprocessor as $1 \mathrm{~K}$ bytes $(\mathrm{K}=1024)$ of randomaccess memory (RAM). The contents of these bytes are formatted into a display of 16 lines by 64 characters and output as video signals. Both text and graphics characters are included in the character set, which is shown in Figure 2. One such interface is required for each video screen. The other microcomputer components were selected for compatibility with the video interfaces and with the S-100 bus. All are hobby market items and were purchased at a local computer store. Table 1

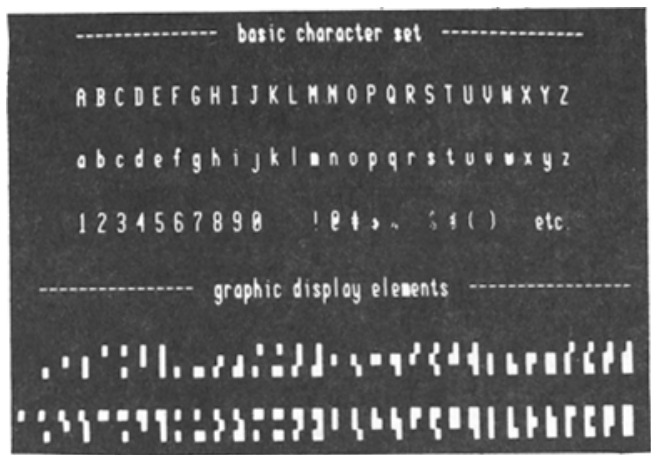

Figure 2. Alphabetic and graphic characters provided by the Polymorphic Systems video terminal interface. Greek letters are also available but are not used in the microcomputer graphics system. 
Table 1

Microcomputer Components and Prices

\begin{tabular}{llll}
\hline \multicolumn{1}{c}{ Description of Item } & \multicolumn{1}{c}{ Vendor } & Model & Price \\
\hline Mainframe* & Vector Graphic & V1-18A & \$95 \\
Z80 CPU board, 2 and 4 MHz & Cromemco & ZPU-W & 395 \\
Memory board, 16K bytes, 250 nsec & MITS & $88-16 \mathrm{~K}$ & 945 \\
ROM board, 8K with programmer & Cromemco & 2708 & 245 \\
Serial and parallel interfaces & Pro. Tech. & 3 P+S & 200 \\
Cassette interface & Tarbell & & 175 \\
Video display boards (6), each & Polymorphic & VTI & 300 \\
Video monitors (6), each & Panasonic & WV-5300 & 225 \\
Keyboard, full ASCII & IMC & KB-1 & 170 \\
Cassette recorder & J. C. Penney & 6536 & 45 \\
Software** & TDL & PKG-1 & 190 \\
Total Hardware/Software Cost & & & $\$ 6,110$ \\
\hline
\end{tabular}

*Includes power supplies and 18-slot mother board.

**Includes $2 \mathrm{~K}$ monitor, text editor, $Z 80$ assembler, $8 \mathrm{~K}$ BASIC, and word processor.

shows the components and the prices in effect at the time of purchase in late 1977. A Z80 processor was chosen for its high speed (4-MHz clock rate) and large instruction set. One instruction, in particular, moves a block of data from one origin to another. This is of importance for moving large amounts of display information into the video interface memories in very short times, so that displays seem to appear instantaneously.

Memory was selected to match the CPU in speed (250-nsec access time). In order to run BASIC language programs together with assembly language subroutines, a size of $16 \mathrm{~K}$ bytes was specified. An additional $4 \mathrm{~K}$ bytes of read-only memory (ROM) were allocated to operating system software, in this case the "Zapple" monitor from Technical Design Labs (TDL). The monitor includes many features needed for efficient software development, including the ability to list the contents of memory, to inspect or change selected memory locations, to store programs on cassette tape, and to control I/O device assignments. The TDL software also includes a program editor, a BASIC interpreter, a Z80 macroassembler, and a word processor.

Because a PDP Lab/8e minicomputer was already in use for data acquisition and analysis in divided-attention studies, it was decided to use the microcomputer system as an intelligent front-end, rather than as a stand-alone device. Accordingly, floppy disk storage was not specified for the microcomputer. Rather, a serial interface was used to permit commands to be transferred from the PDP 8 to the Z80.

\section{IMPLEMENTATION}

The microcomputer components were purchased in kit form. The major hardware effort involved assembling and testing, integrating the various circuit cards in to the Vector mainframe, selecting interface addresses, and loading the Zapple monitor into electronically programmable read-only memory (EPROM). Software development was by far the more complex task, involving de- sign of a symbolic display language and a communication protocol for transmissions between the host PDP 8 and the microcomputer. This section describes these tasks in general terms. A memory map for both the Z80 and PDP 8, showing the location of the component tasks and interfaces, is shown in Figure 3. It should be used for reference throughout this section.

\section{Microcomputer Hardware}

The logical design of the $\mathrm{Z} 80$ microprocessor includes an address space of $64 \mathrm{~K}$ bytes, which are ad dressed as 0000 to FFFF in hexadecimal. The 16K-byte RAM was assigned addresses 0000 through 3FFF This provides for low-memory restart locations (which are accessed when the front panel "reset" button is pressed) and space for the $8 \mathrm{~K}$ BASIC interpreter, plus additional application software which will be discussed in a following section. The 4K-byte EPROM, which contains the monitor, was assigned the highest memory addresses, E000 through FFFF. The video interfaces. each of which appears as $1 \mathrm{~K}$ bytes of RAM, were given
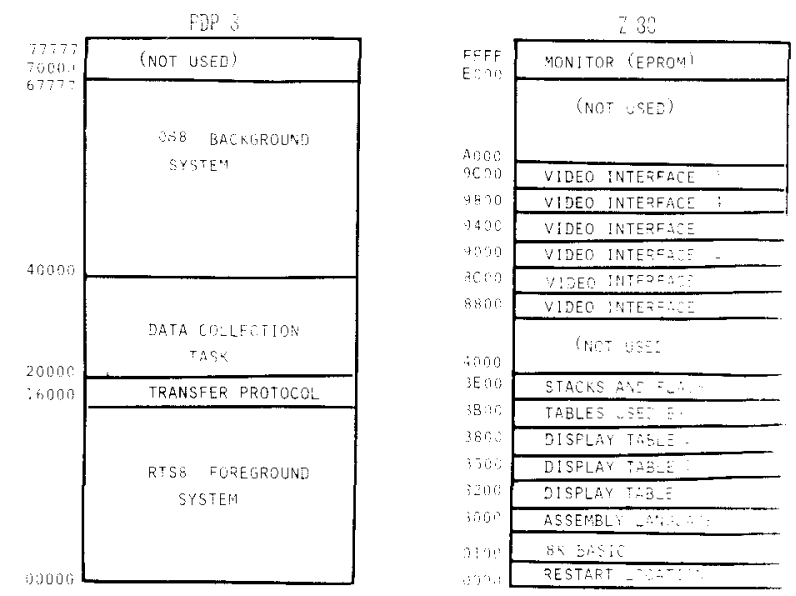

Figure 3. Memory map showing the location of $\mathrm{PDP} \times$ $\mathrm{Z80}$ software components. The six video interfaces are accis as part of the $\mathrm{Z} 80$ memory. 


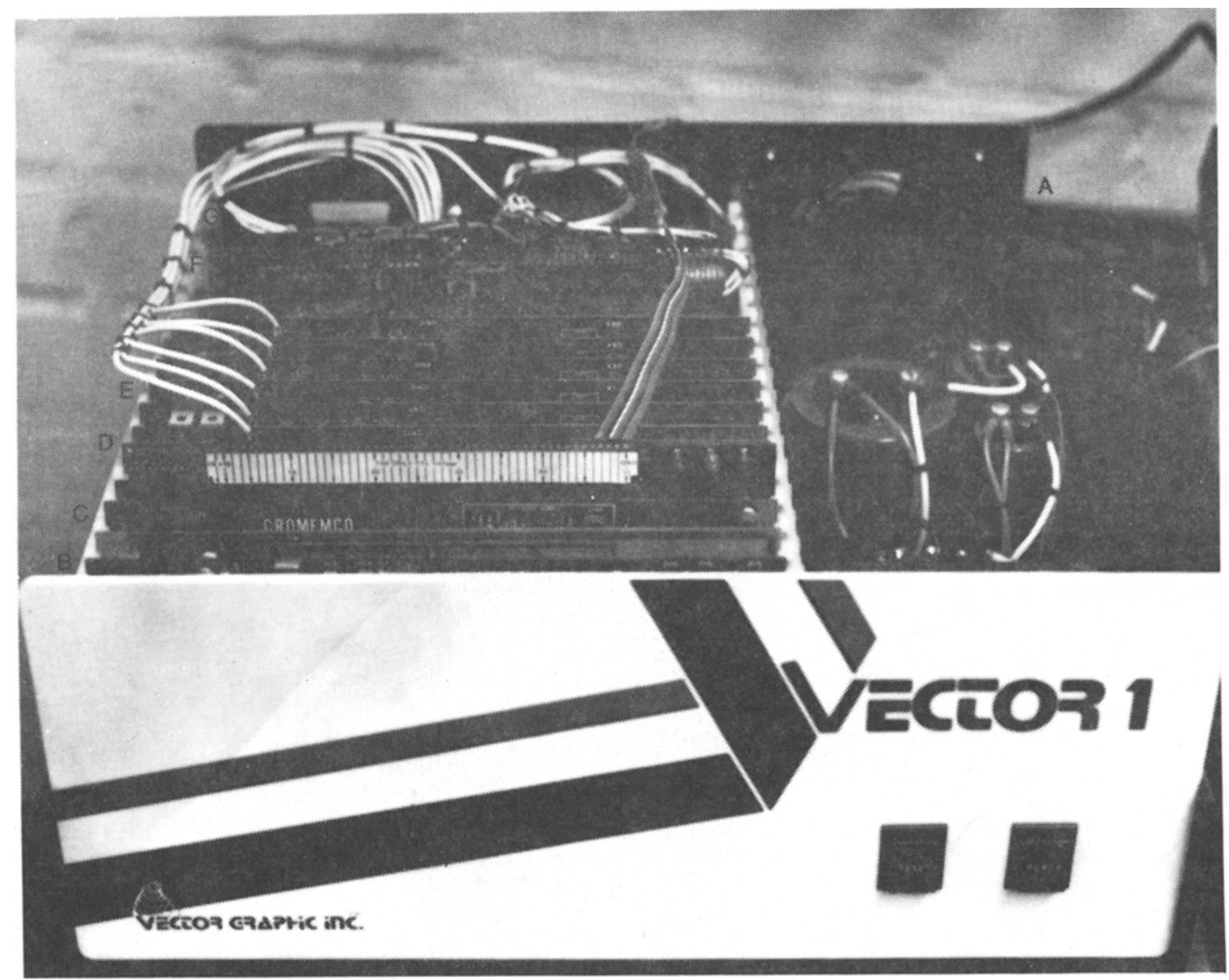

Figure 4. Assembled microcomputer showing (a) main chassis with fan and power supply, (b) Z80 CPU board, (c) ROM board with EPROM programmer, (d) 16K RAM board, (e) video terminal interfaces (6), (f) serial and parallel interfaces, and (g) Tarbell cassette interface.

sequential addresses beginning at 8800 and proceeding in increments of 400 (hexadecimal). This choice was made so that another $16 \mathrm{~K}$ bytes of RAM could be inserted in the future at Address 4000 with no reconfiguration. Also, generous space was allowed for additional video interfaces. If all of the free locations between A000 and E000 were so used, a total of 22 screens could be accessed. The Vector Graphic mainframe with all cards inserted is shown in Figure 4.

\section{Microcomputer Software}

Since the microcomputer system did not include floppy disks, all software was purchased on paper tape. The tapes were transferred to cassette using a handpowered paper-tape reader that was borrowed for the occasion. Subsequent work was done using cassettes for program storage.

Several modifications were made in the TDL monitor so that (1) the operator device driver would access Video Display Interface 0 (at Address 8800) and (2) re- mote motor control and error checking were provided in the Tarbell cassette driver. The monitor was first patched and tested in RAM. Then the updated relocatable code was reloaded into RAM (with suitable address bias) and burned into a 2708 EPROM chip. EPROMs are reprogrammed by ultraviolet erasure and subsequent rewrites.

The remainder of the TDL software was directly loadable from cassette and ran with no modifications or bugs. The bulk of the microcomputer program logic was written in BASIC, since that language is well suited to operator communications. Extensive prompting, input error handling, and character string manipulation can easily be programmed and modified at a later time. Functions such as PEEK and POKE provide access to specific memory locations, and assembly language subroutines may be called to do operations that are time critical or are not easily implemented in BASIC.

The video displays are manipulated by such an assembly language subroutine. The purpose of this 
routine is to load data into one of the three memory areas, called "display tables," and to transfer on command the contents of a selected display table to the appropriate screen interface memory locations. The transfer is done by a sequence of block move instructions, one for each string of data bytes in the selected display table. This permits relatively large picture areas to be defined in a single video frame time.

A self-test mode is provided in which the operator can key in successive bytes of a display command string, such as would normally be transmitted from the host computer. This permits all functions of the display subsystem to be tested without connection to the host.

The complexity of the microcomputer software may be estimated from its size. The BASIC portion requires 104 program statements; the assembly language code occupies 352 bytes. Both of these numbers are expressed in base 10 .

\section{Intercomputer Communications Protocol}

Instructions are sent from the PDP 8 to the $Z 80$ in a concise binary format, using a serial RS232 interface transmitting at 9,600 baud. Each message consists of a header indicating the function to be performed and the length of the message, a string of zero or more data bytes, and a checksum for use in detecting transmission errors. The checksum is the sum, modulo 256 , of all of the message bytes, including the header. The Z80 program logic makes it possible to load the display tables well in advance of the time their contents must be transferred to the video interface memories. The message to trigger a display change is then only 3 bytes in length, permitting a high degree of synchronization between host and microcomputer. Also, for many experiments a lower transmission rate can be used without seriously impacting performance. If necessary, the host computer might be at a remote location, with transmission via acoustic coupler at 300 baud.

\section{PDP 8 Software}

As in the case of the microcomputer, software for the host PDP 8 is written in a high-level language when possible. The majority of program modules are written in standard FORTRAN IV. These include: the translator program which converts symbolic display instructions into binary format, experiment-specific routines to expand concise trial descriptors into sequences of symbolic display instructions, data collection routines, and data analysis routines.

Binary file output in the translator program is accomplished through a library subroutine called WRITEB, which is available from the Digital Equipment Corporation 12-bit users group.

The real-time data collection routines are implemented as a set of tasks in the RTS8 operating system. Their function is to read the binary files produced by the translator program, strip off and interpret certain control information, and transmit the remaining bytes to the microcomputer. Because of the requirements of RTS8, these tasks are written in the PAL8 assembly language.

\section{Symbolic Display Language}

Some of the commands available in the symbolic language are defined in Table 2. These are roughly divided into two classes: those interpreted by the PDP 8 (such as PAUSE) and those resulting in some sequence of bytes being transmitted to the $Z 80$ (such as LOAD, TEXT, and DISPLAY). All command lines are free format, with tokens separated by blanks.

The display tables maintained by the $\mathrm{Z} 80$ are referred to by integers 0,1 , and 2 . The video monitors are referred to as Screens 0, 1, 2, and so on, up to the number implemented (in this case, 5). Screen 0 is reserved for communications with the system operator.

A sequence of instructions generally involved LOADing a display table with data to be displayed, waiting until the appropriate time, and then issuing a DISPLAY command. For example, to clear Screen 1, wait $5 \mathrm{sec}$, and display the message SYSTEM DEMONSTRATION in the center of the screen, the following commands might be used:

\section{CLEAR 1 \\ LOAD 0 \\ ORIGIN 1822 \\ TEXT 'SYSTEM DEMONSTRATION' \\ END \\ PAUSE 50 \\ DISPLAY 0}

Graphics are described by specifying the intensity levels at each desired screen position. As discussed earlier, a screen position may contain either an alphanumeric character or a matrix of three squares vertically by two squares horizontally. Each square element may be either on (bright) or off (dark). An octal digit is used

Table 2

Symbolic Display Commands (Abbreviated List)

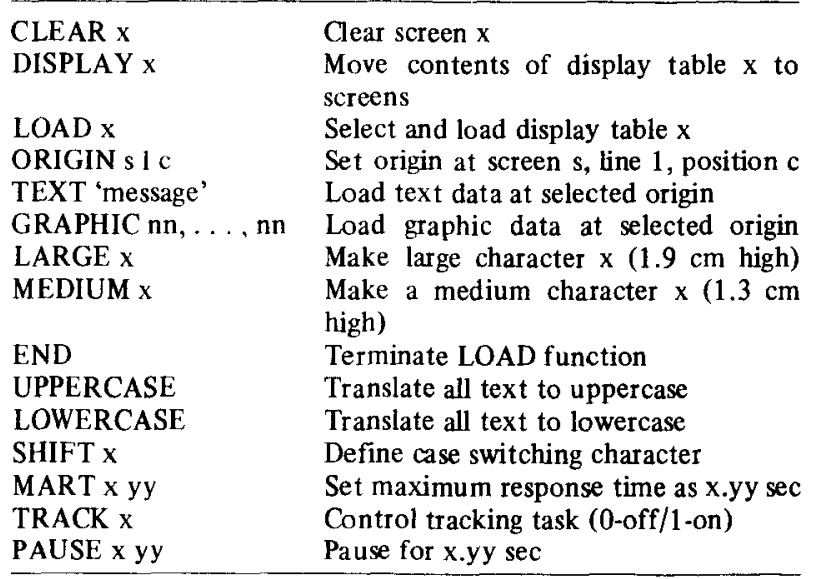


to describe three vertical elements by identifying a 1 bit with each bright element and a 0 bit with each dark element (reading downward). Thus, a code of 73 indicates that in the left column of the character position all three elements are bright, whereas in the right position the top element is dark and the lower two are bright. For example, a bright rectangle at the center of Screen 2, four character positions wide by two lines high, might be programmed in the following way:

CLEAR 2
LOAD 0
ORIGIN 2730
GRAPHIC $77,77,77,77$
ORIGIN 2830
GRAPHIC $77,77,77,77$
END
DISPLAY 0

In many instances, this level of flexibility in dealing with graphic data is not required. Commands are provided for generating large-size characters from simpler graphic elements without the need to specify intensities at the individual screen positions. Thus, to display the digit 9 in large size (eight character positions wide by four lines high) at the center of Screen 3, one might write:

CLEAR 3
LOAD 0
ORIGIN 31028
LARGE 9
END
DISPLAY 0

\section{Experiment Descriptors}

It is often inconvenient to code by hand the symbolic display instructions for an experiment. Several thousand lines of code may be required to describe a reasonably complex experimental sequence. Moreover, several different sequences of trials may be needed to avoid the possibility of order effects in successive runs with the same subject. Because an experimental run usually consists of a number of repeated trials, it is possible to use a preprocessing program to generate sequences of symbolic display instructions tailored to the experiment. In general, the experimenter will hand-code the display instructions for a single trial. Then, in conjunction with a programmer, a command format is defined which includes the parameters that are to vary from trial to trial. The programmer writes a short FORTRAN program (generally on the order of 50 lines) which expands each parameter line into code for one trial. The expansion factor may be considerable, 20 or more symbolic display lines for each input line. The time to write an appropriate preprocessing program is generally about $1 / 2$ day. An alternate strategy is to allow macro definitions within the symbolic display language. As yet, demand has not been great enough to justify implementing this feature.

\section{Data Analysis Software}

Programs for the statistical analysis of response times and tracking performance data are written in FORTRAN IV and run on the PDP 8 under OS8 BATCH. These allow experimental data to be processed immediately after they are collected. Additional experimental data runs may be done while analysis is in progress.

\section{Physical Experiment Setup}

Five video monitors are installed in a $6 \times 6 \mathrm{ft}$ $(1.83 \times 1.83 \mathrm{~m})$ experiment booth made by Industrial Acoustics, together with auxiliary hardware that includes a Burroughs Model BG-12201-2 gas-discharge (electroluminescent) display, a tracking task generator (built in-house), a four-way pushbutton response pad, and a device for recording EPR (Gulf + Western Eye Trac Model 200). Output signals from the tracking task, EPR unit, and the response switches are connected directly to the PDP 8. The tracking task generator, however, is turned on and off by the Z80 using two parallel output lines. The complete system diagram, as installed, is shown in Figure 5.

\section{APPLICATIONS}

The versatility of a microcomputer graphics system is best shown by describing three experiments. No hardware reconfiguration is needed to switch from one

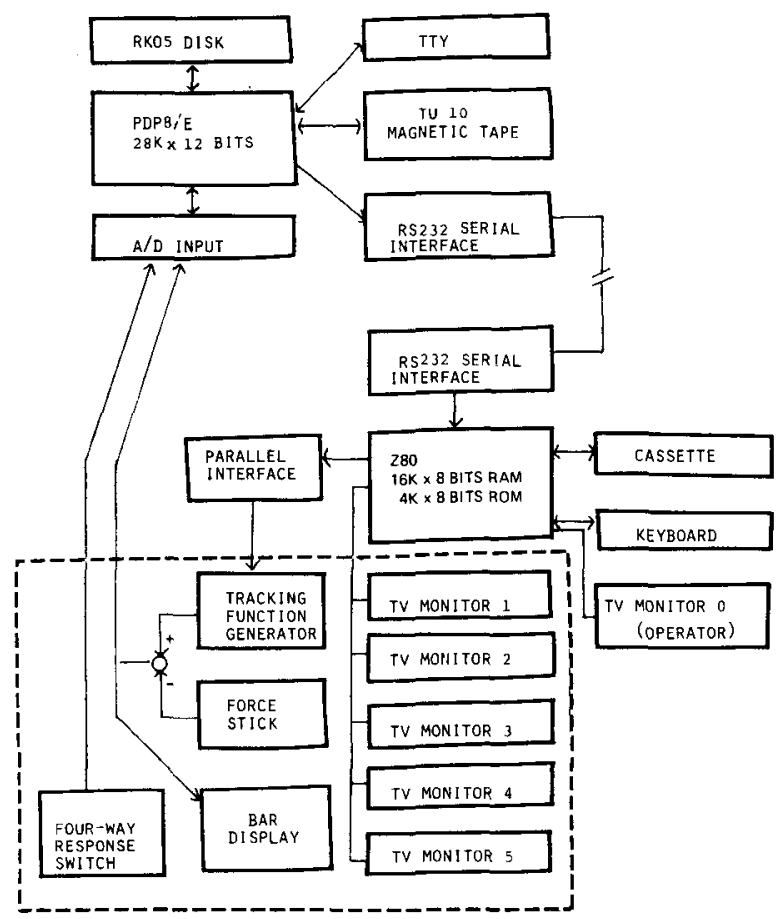

Figure 5. System diagram showing PDP 8 and Z80 hardware components and the data paths connecting them. 
study to another, and, in fact, runs of the three studies are interleaved on a routine basis.

\section{Divided-Attention Study}

This experiment is similar to that performed on the stand-alone SPS (described under Background). The display arc is replaced by Screens $1-4$, each corresponding to one quadrant. At the center of the visual field is an electroluminescent bar display. Instead of LEDs, digits are presented as aggregates of graphic elements. Each digit is $1 / 2$ in. $(1.3 \mathrm{~cm})$ high. The visual angles are the same as those in the earlier experiments.

In terms of throughput requirements, this study is the most complex. Each digit is defined by 32 bytes of data, and there are 24 digit positions. Up to six digits may change every few seconds. In addition, realtime feedback must be provided, so that a correct response will cause a target " 2 " to change to " 0 " within $1 / 4 \mathrm{sec}$. Figure 6 shows a typical video display in this experiment.

\section{Visual Short-Term Memory Test}

This task requires the subject to remember the position of a small rectangle on a plain visual field after turning away to view a distracting task. The distracting task is a sequence of large digits presented at 1 -sec intervals on Screen 5 to the subject's right. The digits must be read aloud; a " 0 " indicates that a response is to be made concerning the position of the stimulus rectangle, displayed on Screen 1 directly in front of the subject. The subject marks on plain paper of the same size as the CRT the position of the stimulus. The response measure is the difference between the target location and response location.

\section{Information Processing Capacity Test}

The object of this test is to measure the ability of the subject to keep track of several things simultaneously. A random sequence of large letters $[1 / 2$ in. $(1.3 \mathrm{~cm})$ in height] is presented at 2 -sec intervals on Screen 1 . The subject's task is to keep track of the number of times

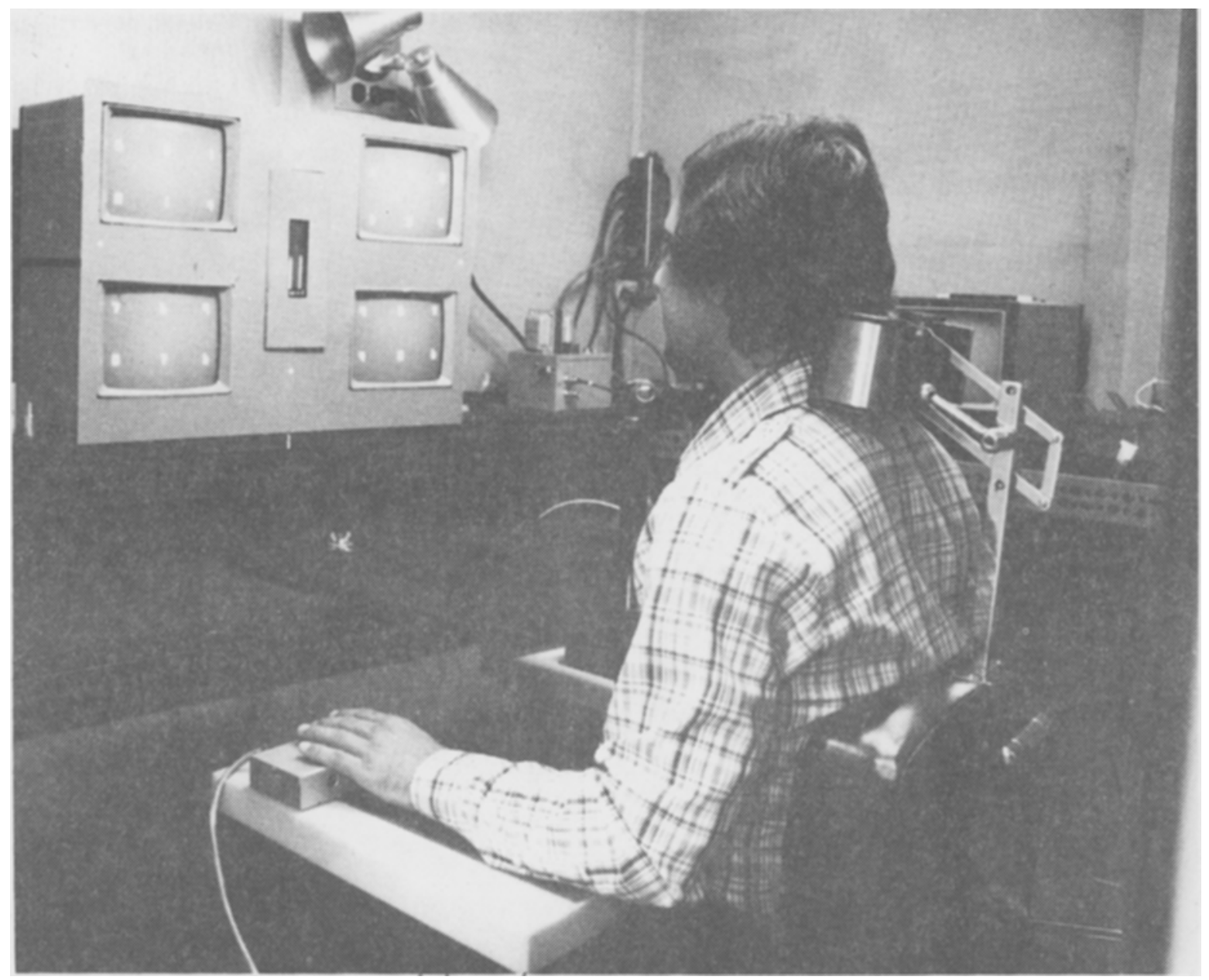

Figure 6. Divided-atten tion experiment using the microcomputer graphics system. 
each letter in a set of target letters appears. Prior to each trial, instructions are displayed on the screen in normal text mode. They indicate the number and identity of the target letters and describe the task to the subject. The number of target letters is increased by one after each trial. Testing continues until either eight targets have been counted simultaneously or the subject elects not to proceed.

\section{CONCLUSIONS}

The microcomputer graphics system has been in operation since March 1978. Only one episode of downtime has been experienced; this occurred during the first month and was diagnosed as a bad chip on one of the video interface boards. Repair was effected in $1 / 2 \mathrm{~h}$. Since then there have been no problems. We estimate the mean time between failures as greater than 6 months, even though no preventive maintenance is done and the system operates in a normal room environment.

Software development costs approximately matched hardware costs. However, if we were building the system today, the total price would be less. Marketplace competition has led to new products with lower prices and greater capabilities, so that the hardware needed to build an identical microcomputer system could be purchased in assembled and tested form (as of November 1978) for $\$ 4,910$. A stand-alone version, using floppy disks for program storage and FORTRAN IV for data analysis, could be built for an additional $\$ 2,500$. A list of these components will be provided on request.

Operator acceptance of the design has been good. Few errors are made, even though several different experiments are run on a tight daily time schedule. The majority of problems seem to involve the electromechanical peripherals on the PDP 8 computer.

Our overall impression is that designing and building this system was not extraordinarily difficult. The methodology of using microcomputer components in place of special-purpose hardware seems to have succeeded very well in meeting the design goals outlined earlier. We hope that our experience will encourage other experimenters to use this approach as well. 